\title{
Anesthesia for a Patient with an Orbital Mass Complicated by Recent Coronary Stenting and Pulmonary Artery Hypertension Due to Langerhans Cell Histiocytosis: the Role of Awake Pulmonary Artery Catheterization
}

\author{
Keita Sato ${ }^{1}$, Brian Rotenberg ${ }^{2}$ and John M Murkin ${ }^{1 *}$ \\ ${ }^{1}$ Department of Anesthesia and Perioperative Medicine, Schulich School of Medicine, The University of Western Ontario, London, Ontario, Canada \\ ${ }^{2}$ Department of Otolaryngology-Head and Neck Surgery, Schulich School of Medicine, The University of Western Ontario, London, Ontario, Canada
}

\section{Introduction}

Pulmonary artery hypertension $(\mathrm{PAH})$ is recognized as one of the most significant risk factors in patients undergoing non-cardiac surgery as it is associated with a variety of increased post-operative morbidities, including respiratory failure, cardiac dysrhythmia, congestive heart failure, renal insufficiency, sepsis, hemodynamic instability and increased mortality $[1,2]$.

Herein we present a patient requiring an urgent short procedure for right frontal sinusectomy, right ethmoidectomy and drainage of right orbital abscess due to histiocytosis infiltration compressing the right ocular nerve in a patient with significant $\mathrm{PAH}$ due to pulmonary Langerhans cell histiocytosis (LCH). The present case was further complicated by recent drug eluting stent (DES) placement and acute withdrawal of clopidogrel therapy [3,4].

$\mathrm{LCH}$ is a rare hystiocytic disorder, which is mainly characterized by organ infiltrations with histiocytosis. Langerhans cell proliferation may involve any organ including bone, lung, hypothalamus/posterior pituitary gland, skin/mucous membranes, lymph nodes, liver, and various soft tissues [5]. Of these, precapillary pulmonary hypertension is common in patients with pulmonary LCH $[6,7]$. Treatment options for PAH with pulmonary LCH include smoking cessation, vasodilator therapies such as endothelin receptor antagonist and phosphodiesterase 5 inhibitor, and lung transplant for end-stage pulmonary LCH [6-8]. We highlight the anesthetic managements for the patient with severe $\mathrm{PAH}$ due to pulmonary $\mathrm{LCH}$ and recent DES.

\section{Case Description}

A 66 year-old monocular male with systemic and pulmonary $\mathrm{LCH}$ and long-standing smoking history on home oxygen therapy presented with acute onset of right-sided headache. Investigations revealed right frontal sinus infiltration compressing the right ocular nerve causing rapidly progressive blindness requiring urgent intervention. His medical history included significant PAH caused by pulmonary $\mathrm{LCH}$, a 50-pack-year smoking history discontinued 10 months previously, obstructive sleep apnea on continuous positive airway pressure (CPAP), recent acute coronary intervention (ACI) followed by drug eluting stent in left main coronary artery (LMCA) and left anterior descending
(LAD) arteries 6 weeks previously, bilateral femoral artery stenosis with claudication, diabetes insipidus on desmopressin caused by histiocytic invasion to pituitary, type 2 diabetes mellitus, and blind left eye due to trauma at the age of 14. Physical examination disclosed a patient on chronic nasal oxygen, potential difficult intubation with grade 3 Mallampati score, and increased antero-posterior chest diameter and distant breath sounds. His medication included home oxygen therapy $3.5 \mathrm{~L} / \mathrm{min}$, tadalafil $40 \mathrm{mg}$, clopidogrel $75 \mathrm{mg}$, aspirin $81 \mathrm{mg}$, diuretics and inhalers for chronic obstructive pulmonary disease (COPD).

On 6-minute walk test 5 months prior, he walked only 420 meters versus a predicted 564 meters with oxygen saturation $79 \%$ and Borg scale of 4. Primary abnormality on pulmonary function test was severe reduction in lung diffusion of $21 \%$ of normal. Concomitant cardiac catheterization demonstrated right atrial pressure $19 \mathrm{mmHg}$, pulmonary artery pressure (PAP) $86 / 44 \mathrm{mmHg}$, pulmonary capillary wedge pressure (PCWP) $12 \mathrm{mmHg}$, cardiac output $4.3 \mathrm{~L} / \mathrm{min}$, pulmonary vascular resistance (PVR) 11.4 Wood units, and mixed venous $\mathrm{O}_{2}$ saturation $55.0 \%$. These results indicate significantly elevated PVR which can predispose to perioperative right ventricular failure and also mandates special anesthetic considerations for preventing intraoperative and postoperative cardiac and respiratory complications. In addition DES to LMCA and LAD 6 weeks previously for which the patient was maintained on dual antiplatelet therapy, significantly increased the risk of surgical site bleeding.

The risk for potential complete blindness due to delay of the surgery versus risk for bleeding in right orbit after immediate surgery were discussed with the otolaryngologists, cardiologists and anesthesiologists. It was decided to withdraw clopidogrel therapy for 10 days prior to procedure with continuance of low dose aspirin to minimize the risk of bleeding and balance potential for DES thrombosis, a delay which also enabled addition of a further anti-PAH

${ }^{\star}$ Correspondence to: John M Murkin, Department of Anesthesia and Perioperative Medicine, Schulich School of Medicine, The University of Western Ontario, London, Ontario, Canada, Tel: (519) 663-3384; Fax: (519) 663-3260; E-mail: john.murkin@lhsc.on.ca

Received: May 02, 2018; Accepted: May 26, 2018; Published: May 29, 2018 
Sato K (2018) Anesthesia for a Patient with an Orbital Mass Complicated by Recent Coronary Stenting and Pulmonary Artery Hypertension Due to Langerhans Cell Histiocytosis: the Role of Awake Pulmonary Artery Catheterization

drug, endothelin receptor antagonist bosentan, to further optimize PVR before procedure.

After discussion with patient and family on the day of surgery including the potential for prolonged postoperative ventilation in Intensive Care Unit, a right radial artery pressure line and pulmonary artery catheter (PAC) were inserted with patient resting comfortably under light sedation. ${ }^{8}$ Surprisingly, initial PAP was $38 / 15 \mathrm{mmHg}$ which was much lower than expected and raised the possibility of early extubation. Induction and intubation were conducted uneventfully with fentanyl $100 \mathrm{mcg}$, propofol $70 \mathrm{mg}$ and rocuronium $40 \mathrm{mg}$ using video-assisted laryngoscopy. Anesthesia was maintained with end-tidal sevoflurane $1.0-1.3 \%$ and remifentanil $0.05 \mathrm{mcg} / \mathrm{kg} / \mathrm{min}$.

After induction of anesthesia positive pressure ventilation was started maintaining $\mathrm{SpO}_{2}$ levels of $99-100 \%$ at low tidal volume (Vt $5-6 \mathrm{ml} / \mathrm{kg}$ ) and low positive end expiratory pressure (PEEP $<5 \mathrm{~cm}$ $\left.\mathrm{H}_{2} \mathrm{O}\right)$ with end-tidal $\mathrm{CO}_{2}\left(\mathrm{ETCO}_{2}\right)$ levels of $30-35 \mathrm{mmHg}$. Shortly after induction PAP rose rapidly and progressively in excess of $71 / 42 \mathrm{mmHg}$, treated with a milrinone bolus of $0.5 \mathrm{mg}$, followed by continuous infusion of $0.125 \mathrm{mcg} / \mathrm{kg} / \mathrm{min}$ and nitroglycerin infusion of $30-60 \mathrm{mcg} / \mathrm{min}$ were employed as pulmonary vasodilators. Meanwhile, systolic blood pressure dropped to $<100 \mathrm{mmHg}$, and was treated with continuous infusion of norepinephrine of $2-3 \mathrm{mcg} /$ min and intermittent bolus of vasopressin of 1-2 units to maintain blood pressure. Avoidance of hypercarbia was a specific goal with maintenance of low $\mathrm{ETCO}_{2}$, however rising PAP prompted STAT arterial gas analysis which revealed $\mathrm{PaCO}_{2}$ level of $53 \mathrm{mmHg}$, which was corrected by increasing minute ventilation. After these treatments, pulmonary artery pressure progressively decreased to $55 / 25 \mathrm{mmHg}$. As anticipated, surgical drainage of frontal and orbital abscess was completed within one hour and due to further normalization of PAP, vasoactive medications were progressively discontinued and the patient was successfully extubated in the operation room. The patient was observed uneventfully overnight in the intensive care unit without vasoactive medications and discharged from hospital without any adverse outcome on postoperative day 2 with resumption of dual antiplatelet therapy. Histological analysis confirmed inflammatory abscess tissue without $\mathrm{LCH}$ infiltration and the patient's vision rapidly recovered and he was doing well at 6 week follow up visit.

\section{Discussion}

Minor surgery for patients with severe comorbidities is often problematic for anesthesiologists. In this case, the surgery was relatively short and minimally invasive; on the other hand, the patient had recent DES and significant PAH. PAH is a well-known risk factor for mortality and morbidity after non-cardiac surgery, and perioperative management is well established [9-11]. However, in addition to PAH, this case was also complicated by acute ongoing symptoms potentially leading to total visual loss in the presence of dual antiplatelet therapy related to recent DES deployment. Multidisciplinary consultation considered the consequences of delaying the surgical procedure versus the increased risk of perioperative bleeding all balanced against risk of in-stent thrombosis. Bleeding exacerbated by dual anti-platelet therapy would be highly challenging after surgery since the optical nerve involved by the lesion was confined in the closed space of the right orbit. It was considered that a delay of the procedure would be permitted considering the slow growing property of LCH so that a 10 days cessation period for clopidogrel with continuance of aspirin would reduce the risk of stent thrombosis, consistent with the management strategy of antiplatelet therapy in the current non-cardiac surgery guidelines [12,13]. Platelet function testing was not available although this may have been beneficial to better determine the optimal timing of urgent surgery following cessation of antiplatelet therapy [14].

For the anesthetic plan, usage of specific anesthetic agents seems to be relatively unimportant as various anesthetics and techniques have been successfully employed for patients with PAH [15]. However, in light of the relative normalization of pre-induction PAP and considering the potential disadvantages of postoperative ventilation, we chose sevoflurane and remifentanil-based balanced anesthesia thus avoiding high dose opioid and maintaining the option of rapid extubation at end of surgery.

Transesophageal echocardiography (TEE) is widely used during cardiac and non-cardiac surgery for patients with the compromised cardiac function and has the advantage of being less invasive than PAC. TEE also provides direct information of right ventricular size and function in addition to estimating right ventricular systolic pressure from tricuspid regurgitation jet. However, TEE cannot readily be employed during induction of anesthesia and requirement for surgical access via transnasal route could significantly limit its intraoperative usage.

Our pre-induction usage of PAC facilitated the immediate assessment of PAP which detected much lower resting PAP than reported previously. This new information profoundly impacted both the anesthetic plan and the proposed postoperative management, as has been recently discussed [8]. Additionally, discriminating between elevated PAP due to myocardial ischemia versus pulmonary vasoconstriction was readily achieved with $\mathrm{PAC}$ which provided not only continuous monitoring of PAP but also enabled measurement of PCWP, which remained low $(7-8 \mathrm{mmHg})$ at all times and indicated increasing PVR rather than myocardial ischemia as cause of PAH and prompted institution of vasodilator therapy as well as modification of ventilation settings to achieve normocarbia.

The exacerbation of PAH after induction of general anesthesia was caused by high $\mathrm{PaCO}_{2}$ and the direct negative impact of positive pressure ventilation on PVR despite usage of low Vt and low PEEP and low $\mathrm{ETCO}_{2}$ [16]. The heterogeneity of ventilation associated with COPD [17] is one of the causes of variably increased arterial and endtidal $\mathrm{CO}_{2}$ difference which made end-tidal $\mathrm{CO}_{2}$ monitoring grossly inaccurate in this patient. PAP monitoring was thus highly beneficial in immediately detecting the alteration of PVR and encouraging us to interrogate the cause and remediate the elevated $\mathrm{PaCO}_{2}$.

The current guidelines recommend at least 180 days interval for elective non-cardiac surgery after placing DES $[3,13]$. This is because of the risk of in-stent thrombosis and higher rates of mortality and morbidity in patients with recent DES deployment undergoing noncardiac surgery $[3,4,12]$. In the present case, the right ocular nerve was compressed by tumor which required urgent surgery to avoid total vision loss and the surgery was undertaken 6 weeks after the DES implantation. To balance the risks of in-stent thrombosis versus catastrophic bleeding or hematoma at the surgical site low dose of aspirin was continued while further clopidogrel was withheld, consistent with the current guideline [13].

To our knowledge, there are no reports in the literatures detailing anesthetic strategies for patients with $\mathrm{PAH}$ due to $\mathrm{LCH}$. Multidisciplinary-team decision making was essential in determining appropriate procedural timing, the management of DES antiplatelet therapy, and optimizing the treatment regimen for $\mathrm{PAH}$, and contributed greatly to the uneventful course of this patient. 
This case highlights the possible comorbidities of LHC and anesthetic considerations. Of these, PAH should be suspected if the lung is infiltrated by histiocytosis, and will require close monitoring of right ventricular function and PVR, and ready availability of potential treatment options including inotropes and vasodilators. Of particular note, positive pressure ventilation had a dramatic impact on PAH and end-tidal $\mathrm{CO}_{2}$ was grossly misleading and inaccurate in determining $\mathrm{PaCO}_{2}$. The pre-induction insertion of PAC provided critical new information on basal pulmonary artery pressure and enabled early detection of increasing PAH during surgery, which was beneficial not only for maintenance of hemodynamic during surgery but also for determining extubation strategy at the end of surgery.

\section{References}

1. Ramakrishna G, Sprung J, Ravi BS, Chandrasekaran K, McGoon, et al. (2005) Impact of pulmonary hypertension on the outcomes of noncardiac surgery: predictors of perioperative morbidity and mortality. J Am Coll Cardiol 45: 1691-1699. [Crossref]

2. Kaw R, Pasupuleti V, Deshpande A, Hamieh T, Walker E, et al. (2011) Pulmonary hypertension: an important predictor of outcomes in patients undergoing non-cardiac surgery. Respir Med 105: 619-624. [Crossref]

3. Wijeysundera DN, Wijeysundera HC, Yun L, Wąsowicz M, Beattie WS, et al. (2012) Risk of elective major noncardiac surgery after coronary stent insertion: a populationbased study. Circulation 126: 1355-1362. [Crossref]

4. Hawn MT, Graham LA, Richman JS, Itani KM, Henderson WG, et al. (2013) Risk of major adverse cardiac events following noncardiac surgery in patients with coronary stents. JAMA 310: 1462-1472. [Crossref]

5. Howarth DM, Gilchrist GS, Mullan BP, Wiseman GA, Edmonson JH, et al. (1999) Langerhans cell histiocytosis: diagnosis, natural history, management, and outcome. Cancer 85: 2278-2290.

6. Le Pavec J, Lorillon G, Jais X, Tcherakian C, Feuillet S, et al. (2012) Pulmonary Langerhans cell histiocytosis-associated pulmonary hypertension: clinical characteristics and impact of pulmonary arterial hypertension therapies. Chest 142: 1150-1157. [Crossref]
7. Dauriat G, Mal H, Thabut G, Mornex JF, Bertocchi M, et al. (2006) Lung transplantation for pulmonary langerhans' cell histiocytosis: a multicenter analysis. Transplantation 81: 746-750. [Crossref]

8. Hargrave J (2017) Con: Preinduction Pulmonary Artery Catheter Placement Is Advisable in Patients With Right Ventricular Dysfunction Secondary to Severe Pulmonary Hypertension. J Cardiothorac Vasc Anesth 31: 1514-1518. [Crossref]

9. Fox DL, Stream AR, Bull T (2014) Perioperative management of the patient with pulmonary hypertension. Chest 18: 310-318. [Crossref]

10. Hosseinian L (2014) Pulmonary hypertension and noncardiac surgery: implications for the anesthesiologist. J Cardiothorac Vasc Anesth 28: 1064-1074. [Crossref]

11. Pilkington SA, Taboada D, Martinez G (2015) Pulmonary hypertension and its management in patients undergoing non-cardiac surgery. Anaesthesia 70: 56-70. [Crossref]

12. Fleisher LA, Fleischmann KE, Auerbach AD, Barnason SA, Beckman JA, et al (2014) 2014 ACC/AHA guideline on perioperative cardiovascular evaluation and management of patients undergoing noncardiac surgery: a report of the American College of Cardiology/American Heart Association Task Force on Practice Guidelines. Circulation 130: e278-333. [Crossref]

13. Valgimigli M, Bueno H, Byrne RA et al. 2017 ESC focused update on dual antiplatelet therapy in coronary artery disease developed in collaboration with EACTS: The Task Force for dual antiplatelet therapy in coronary artery disease of the European Society of Cardiology (ESC) and of the European Association for Cardio-Thoracic Surgery (EACTS). Eur Heart J 39: 213-260. [Crossref]

14. Orme R, Judge HM, Storey RF (2017) Monitoring Antiplatelet Therapy. Semin Thromb Hemost 43: 311-319. [Crossref]

15. Pritts CD, Pearl RG (2010) Anesthesia for patients with pulmonary hypertension. Curr Opin Anaesthesiol 23: 411-416. [Crossref]

16. Zamanian RT, Haddad F, Doyle RL, Weinacker AB (2007) Management strategies for patients with pulmonary hypertension in the intensive care unit. Crit Care Med 35 2037-2050.

17. Kaczka DW, Lutchen KR, Hantos Z (2011) Emergent behavior of regional heterogeneity in the lung and its effects on respiratory impedance. J Appl Physiol (1985) 110: 14731481. [Crossref]

Copyright: (C2018 Sato K. This is an open-access article distributed under the terms of the Creative Commons Attribution License, which permits unrestricted use, distribution, and reproduction in any medium, provided the original author and source are credited. 